\title{
A Large Multicenter Prospective Study of Community- Onset Healthcare Associated Bacteremic Urinary Tract Infections in the Era of Multidrug Resistance: Even Worse than Hospital Acquired Infections?
}

\author{
Silvia Gómez-Zorrilla (D) · Federico Becerra-Aparicio • Inmaculada López Montesinos • Enrique Ruiz de Gopegui • \\ Inmaculada Grau • Vicente Pintado · Belén Padilla • Natividad Benito · Lucía Boix-Palop · Maria Carmen Fariñas • \\ María Peñaranda · Maria Rocío Gamallo · Jose Antonio Martinez • Elena Morte-Romea · Jose Luis Del Pozo • \\ Xavier Durán-Jordá · Jazmin Díaz-Regañón · Diego López-Mendoza · Rafael Cantón · Antonio Oliver · \\ Patricia Ruiz-Garbajosa · Juan Pablo Horcajada
}

Received: July 11, 2021 / Accepted: September 14, 2021 / Published online: October 9, 2021

(c) The Author(s) 2021

\section{ABSTRACT}

Introduction: Healthcare-associated (HCA) infections represent a growing public health problem. The aim of this study was to compare community-onset healthcare associated (COHCA) bacteremic urinary tract infections (BUTI)

The members of REIPI/GEIRAS-GEMARA SEIMC ITUBRAS-2 Group are listed in Acknowledgements.

Supplementary Information The online version contains supplementary material available at https:// doi.org/10.1007/s40121-021-00537-0.

S. Gómez-Zorrilla $(\bowtie)$ · I. López Montesinos · J. P. Horcajada ( $\square)$ Infectious Diseases Service, Hospital del Mar, Infectious Pathology and Antimicrobials Research Group (IPAR), Institut Hospital del Mar d'Investigacions Mèdiques (IMIM), Universitat Pompeu Fabra, Passeig Marítim 25-27, 08003 Barcelona, Spain e-mail: sgomezzorrilla@parcdesalutmar.cat J. P. Horcajada e-mail: jhorcajada@psmar.cat

S. Gómez-Zorrilla · F. Becerra-Aparicio · I. López Montesinos · E. Ruiz de Gopegui · I. Grau · V. Pintado $\cdot$ B. Padilla $\cdot$ M. C. Fariñas . J. A. Martinez · J. L. Del Pozo · R. Cantón · A. Oliver . P. Ruiz-Garbajosa · J. P. Horcajada Red Española de Investigación en Patología Infecciosa (REIPI), Instituto de Salud Carlos III, Madrid, Spain and hospital-acquired (HA)-BUTI with special focus on multidrug resistances (MDR) and outcomes.

Methods: ITUBRAS-project is a prospective multicenter cohort study of patients with HCABUTI. All consecutive hospitalized adult patients with CO-HCA-BUTI or HA-BUTI episode were included in the study. Exclusion criteria were: patients $<18$ years old, nonhospitalized patients, bacteremia from another source or primary bacteremia, non-healthcarerelated infections and infections caused by unusual pathogens of the urinary tract. The

F. Becerra-Aparicio - R. Cantón · P. Ruiz-Garbajosa Servicio de Microbiología, Hospital Universitario Ramón y Cajal and Instituto Ramón y Cajal de Investigación Sanitaria (IRYCIS), Madrid, Spain

E. Ruiz de Gopegui · A. Oliver

Microbiology Service, Hospital Universitari Son Espases, Palma de Mallorca, Spain

I. Grau

Infectious Diseases Service, Hospital Universitari de Bellvitge, Barcelona, Spain

V. Pintado

Infectious Diseases Service, Hospital Universitario Ramón y Cajal, Madrid, Spain

B. Padilla

Infectious Diseases Service, Hospital Universitario

Gregorio Marañón, Madrid, Spain 
main outcome variable was 30-day all-cause mortality with day 1 as the first day of positive blood culture. Logistic regression was used to analyze factors associated with clinical cure at hospital discharge and with receiving inappropriate initial antibiotic treatment. Cox regression was used to evaluate 30-day all-cause mortality.

Results: Four hundred forty-three episodes were included, 223 CO-HCA-BUTI. Patients with CO-HCA-BUTI were older $(p<0.001)$ and had more underlying diseases $(p=0.029)$ than those with HA-BUTI. The severity of the acute illness (Pitt score) was also higher in CO-HCABUTI $(p=0.026)$. Overall, a very high rate of MDR profiles $(271 / 443,61.2 \%)$ was observed, with no statistical differences between groups. In multivariable analysis, inadequate empirical treatment was associated with MDR profile (aOR 3.35; 95\% CI 1.77-6.35), Pseudomonas aeruginosa (aOR 2.86; 95\% CI 1.27-6.44) and Charl- son index (aOR 1.11; 95\% CI 1.01-1.23). Mortality was not associated with the site of acquisition of the infection or the presence of MDR profile. However, in the logistic regression analyses patients with CO-HCA-BUTI (aOR $0.61 ; 95 \%$ CI $0.40-0.93$ ) were less likely to present clinical cure.

Conclusion: The rate of MDR infections was worryingly high in our study. No differences in MDR rates were found between CO-HCA-BUTI and HA-BUTI, in the probability of receiving inappropriate empirical treatment or in 30-day mortality. However, CO-HCA-BUTIs were associated with worse clinical cure.

Keywords: Urinary tract infections; Bloodstream infections; Multidrug resistant; Community-onset healthcare-associated infections; Hospital-acquired infections

\author{
N. Benito \\ Infectious Diseases Service, Hospital de la Santa \\ Creui Sant Pau-Institut d'Investigació Biomèdica \\ Sant Pau, Universitat Autònoma de Barcelona, \\ Barcelona, Spain \\ L. Boix-Palop \\ Infectious Diseases Service, Hospital Mutua de \\ Terrassa, Barcelona, Spain \\ M. C. Fariñas \\ Infectious Diseases Service, Hospital Universitario \\ Marqués de Valdecilla, Santander, Spain \\ M. Peñaranda \\ Infectious Diseases Service, Hospital Universitari \\ Son Espases, Palma de Mallorca, Spain \\ M. R. Gamallo \\ Infectious Diseases Service, Complexo Hospitalario \\ Universitario de Pontevedra, Pontevedra, Spain
}

\author{
J. A. Martinez \\ Infectious Diseases Service, Hospital Clinic, \\ Barcelona, Spain \\ E. Morte-Romea \\ Infectious Diseases Service, Hospital Clínico \\ Universitario "Lozano Blesa", Zaragoza, Spain \\ J. L. Del Pozo \\ Infectious Diseases Service, Clínica Universidad de \\ Navarra, Pamplona, Spain \\ X. Durán-Jordá \\ Methodology and Biostatistics Support Unit, \\ Institut Hospital del Mar d'Investigacions Mèdiques \\ (IMIM), Barcelona, Spain \\ J. Díaz-Regañón · D. López-Mendoza \\ Medical Department, MSD Spain, Madrid, Spain
}




\section{Key Summary Points}

Healthcare-associated (HCA) infections represent a growing public health problem.

Community-onset healthcare associated infections are increasing in complexity, but little is known about whether they should be managed as hospital-acquired infections.

We compared hospital-acquired and community-onset HCA bacteremic urinary tract infections (UTIs) with special focus on multidrug resistance (MDR) and clinical outcomes.

Community-onset HCA bacteremic UTIs occurred in eldery and sicker patients and were associated with a worse clinical cure at hospital discharge than hospitalacquired infections.

No differences were found in MDR rates or in mortality between groups.

\section{INTRODUCTION}

Urinary tract infections (UTIs) are among the three most frequent healthcare-associated (HCA) infections following respiratory and surgical site infections $[1,2]$. They are one of the most common reasons for prescribing antibiotics, with a substantial important economic burden [3].

Antimicrobial resistance represents a growing public health issue with significant impact on antibiotic use and patient outcomes [3-5]. In recent years, there has been an alarming increase in antimicrobial resistance among uropathogens [3]. Infections caused by extended-spectrum $\beta$-lactamase (ESBL), AmpC $\beta$-lactamase- and carbapenemase-producing Enterobacterales are increasing worldwide [3, 4, 6, 7]. Most of the ESBL-producing Enterobacterales infections appear after healthcare exposure [6, 8]. Carbapenem-resistant infections have increased in the setting of community-acquired or community-onset infections, with a prevalence ranging from 0.04 to $29.5 \%$ [9], while multidrug-resistant (MDR) and extensively drug-resistant (XDR) Pseudomonas aeruginosa isolates have disseminated globally with increased rates in recent years $[5,10]$.

HCA infections include both community onset healthcare-associated (CO-HCA) and hospital-acquired (HA) infections [11, 12]. COHCA infections involve a heterogeneous group of patients, such as long-term care facility patients, hemodialysis patients or outpatients receiving intravenous chemotherapy [12]. These infections occur in more complex patients and are associated with worse outcomes than community-acquired infections [13-16]. Nevertheless, little is known about whether CO-HCA infections behave differently from those acquired in the hospital setting [17].

Given the importance of UTI in terms of prevalence, emergence of MDR isolates and the complexity of HCA infections, it is interesting to investigate UTI in the HCA setting. In 2010 our group conducted a multicenter prospective study [18] evaluating bacteremic urinary tract infections (BUTI). It suggested that CO-HCABUTIs were more like HA-BUTIs than community-acquired group. Approaching CO-HCA carefully and considering their complexity should be crucial to improve their management. In the present study, we aimed to compare CO-HCA-BUTI and HA-BUTI with special focus on the influence of MDR and clinical outcomes. We hypothesized that CO-HCA-BUTI would be similar to HA-BUTI in terms of clinical outcomes and MDR rates.

\section{METHODS}

\section{Study Design and Study Population}

This study is part of the ITUBRAS-2 project, which aimed to describe the clinical and microbiological characteristics and outcomes of HCA-BUTIs. A prospective, observational, multicenter cohort study was carried out at 12 university hospitals in the framework of the 
Spanish Network for Research in Infectious Diseases (http://www.reipi.org). The patient recruitment was carried out between August 2017 and April 2019.

Adult patients who met Friedman's criteria [12] for CO-HCA-BUTI or HA-BUTI were included. A prospective review of all patients with potential uropathogen isolated in blood cultures at each hospital was conducted. The following were considered as potential uropathogens: Enterobacterales, $P$. aeruginosa, other non-fermenting gram-negative bacilli, Staphylococcus saprophyticus, Streptococcus agalactiae and Enterococcus spp. Exclusion criteria were: patients $<18$ years old, non-hospitalized patients, bacteremia from another source or primary bacteremia, non-healthcare-related infections and infections caused by unusual pathogens of the urinary tract. Polymicrobial infections were included.

The information was entered into a specifically designed electronic research database. All cases were reviewed by a Contract Research Organization (ADKOMA-CRO) and one of the authors (SG-Z), and if controversy was found a double-check was performed by the investigator team at each hospital. The study was approved by the Clinical Research Ethical Committee of Hospital del Mar (registration number 2016/6957/I) and by the local Ethics Committees of each institution (supplementary material). All patients provided written informed consent at the screening. If patients were unable to provide informed consent, they could be entered with the signature of a relative or legal representative. The study was conducted in accordance with the International Conference on Harmonization Good Clinical Practice Guideline and with the 1964 Helsinki Declaration and its later amendments or comparable ethical standards.

\section{Variables and Definitions}

BUTI was considered when the presence of one or more uropathogens in blood cultures was associated with urinary tract symptoms or asymptomatic UTI with the isolation of the same uropathogen in a urine culture and in absence of another source of infection.

CO-HCA-BUTI was defined when the episode was detected at hospital admission or within the first $48 \mathrm{~h}$, which fulfilled any of the following Friedman criteria [12]: (1) receiving intravenous therapy, wound care or specialized nursing care at home by qualified healthcare workers within 30 days prior the episode; (2) attending a hospital, hemodialysis ward or receiving intravenous chemotherapy within 30 days; (3) being hospitalized in an acute care hospital for $\geq 2$ days within 90 days; (4) residing in a nursing home or long-term care facility; (5) being subject to an invasive urinary procedure within 30 days of the episode or having a longterm indwelling urethral catheter. HA-BUTI was considered when bacteremia occurred beyond $48 \mathrm{~h}$ of hospital admission.

Demographic and clinical characteristics of patients were recorded prospectively. Gender, age, chronic conditions, severity of underlying diseases calculated using the Charlson comorbidity index [19] and the McCabe [20] score were recorded. Patients were considered immunosuppressed if chemotherapy, radiotherapy, corticosteroids and/or other immunosuppressive drugs were administered within the 3 prior months to the bacteremia. Previous urologic history was recorded. Previous use of antibiotic was defined as $>48 \mathrm{~h}$ of antibiotic treatment in the 3 months prior to the infection. The severity of acute illness at presentation was calculated using the Pitt bacteremia score [21]. Antimicrobial therapy was considered adequate when the blood culture isolate was susceptible to at least one of the antimicrobial agents prescribed and the dose was in accordance with the current medical standards.

\section{Outcome and Follow-Up}

The main outcome variable was 30-day all-cause mortality with day 1 as the first day of positive blood culture. Secondary outcomes were clinical response at hospital discharge, length of hospital stay and rate of inadequate empirical antibiotic treatment. The main variable evaluated was the site of acquisition of the infection 
(CO-HCA or HA). Clinical assessment was evaluated $48-72 \mathrm{~h}$ after the bacteremia and at hospital discharge. Clinical stability at $48-72 \mathrm{~h}$ was considered when the patient was afebrile and with no persistence of signs/symptoms of sepsis attributable to bacteremia at $48-72 \mathrm{~h}$. Clinical cure at hospital discharge was considered when all signs and symptoms of infection were completely resolved at hospital discharge. Clinical improvement was considered when the patient improved but with persistence or recurrence of any signs or symptoms at hospital discharge. Length of hospital stay was defined as stay (days) from the onset of the bacteremia (first day of positive blood culture) until the hospital discharge. Microbiological follow-up was conducted with control blood cultures in case of persistence of fever $>48-72 \mathrm{~h}$ after the bacteremia. All patients were followed for 30 days.

\section{Microbiological Studies}

All isolates recovered from blood samples were sent to the reference laboratories (Ramón y Cajal, Son Espases), where bacterial identification was confirmed by MALDI-TOF MS (Bruker Daltonics, Bremen, Germany). Antimicrobial susceptibility was studied using the standard broth microdilution method [22]. We defined susceptible isolates combining those categorized as $S$ (susceptible, standard dose regimen) and I (susceptible, increased exposure) according to EUCAST-2019 criteria (http://www. eucast.org/clinical_breakpoints/). The following agents were tested: ampicillin, amoxicillinclavulanate, piperacillin-tazobactam, ceftazidime, cefotaxime, cefepime, ceftolozane-tazobactam, aztreonam, imipenem, meropenem, ciprofloxacin, gentamicin, tobramycin, amikacin, colistin, fosfomycin, trimethoprim/sulfamethoxazole and vancomycin.

Multidrug-resistant profile was defined in accordance with international definitions [23]. MDR was defined as a strain non-susceptible to at least one agent in $\geq 3$ antimicrobial categories. XDR was defined as non-susceptibility to at least one agent in all but $\leq 2$ categories. Pandrug resistance (PDR) was defined as resistant to all antimicrobial agents.
ESBL production was screened by the doubledisk synergy method and/or the ROSCO ESBL + AMPc Confirm Kit (ROSCO Diagnostica, Taastrup, Denmark) $[24,25]$. The screening of carbapenemase production included the colorimetric Carba-NP test (bioMérieux, La Balmeles-Grottes, France) and assessment of the inhibition-based profile using the ROSCO KPC/ Metallo- $\beta$-lactamase (MBL) and OXA-48 Confirm Kit (ROSCO Diagnostica, Taastrup, Denmark) $[26,27]$. The presence of horizontally acquired $\beta$-lactamases in $P$. aeruginosa was also screened through the ceftazidime/imipenem cloxacillin-inhibition test and the presence of MBLs confirmed by the imipenem/meropenem EDTA double-disk synergy test as described previously [28].

\section{Statistical Analysis}

Determining the sample size required (218 patients in each arm) was based on the results of a previous study [18] and in order to detect a $15 \%$ difference in the proportion of 30-day mortality between groups, using correction for continuity with a significance level of $95 \%$ and power of $80 \%$ and considering a patient loss of $10 \%$. Moreover, a post hoc power calculation was conducted regarding the clinical cure as outcome. Accepting an alpha risk of 0.05 in a two-sided test with 223 subjects in the COHCA-BUTI group and 220 in the HA-BUTI, the statistical power was $74 \%$ to recognize as statistically significant the difference in proportions of MDR profile from $53.4 \%$ in the first group to $65.5 \%$ in the second group.

Categorical variables were presented as number of cases and percentages and continuous variables as median and interquartile range (IQR). Comparative analyses were performed with $X^{2}$ or Fisher's test for categorical variables and the Mann-Whitney $U$ test for continuous variables, as appropriate. A logistic regression model was used for examining independent variables associated with (1) clinical cure and (2) receiving inappropriate empirical treatment. A bivariate analysis was performed to select potential variables that might affect the response. Variables with a $p$ value $<0.10$ were 
included in the model as well as those not statistically significant but clinically relevant. Resistance profile and appropriate empirical treatment were considered clinically relevant variables to be forced in the regression model as they may impact clinical outcomes [5, 29-32]. Then, the logistic regression model was built using a stepwise automatic variable selection procedure. Results were expressed as odds ratio (OR) and 95\% confidence interval (95\% CI). Regarding 30-day mortality, we used a multivariable Cox regression model. Results were reported as hazard ratio (HR) and 95\% CI. Multivariable analysis included variables with $p$ value $<0.10$ in the unadjusted analysis and/or those clinically relevant. Age was considered a clinically relevant variable as clearly impacting mortality. The site of acquisition of infection was also forced in the multivariable analysis as it was the aggrupation variable. Collinearity diagnostics were applied by controlling the variance inflation factor (VIF). Moreover, for Cox regressions, the proportional hazard assumption, checked by examining Schoenfeld residuals (for overall model and variable by variable), was not violated. All analyses were two-tailed, and $p<0.05$ was considered statistically significant. Statistical analyses were carried out using STATA.15 software (StataCorp. 2017. Stata Statistical Software: Release 15. College Station, TX: StataCorp LLC).

\section{RESULTS}

\section{Clinical Features According to Site of Acquisition of the Infection}

A total of 443 episodes of HCA-BUTI were included. Of these, 223 were CO-HCA-BUTI while 220 were HA-BUTI. Table 1 shows a comparative analysis of baseline data according to the site of acquisition of the infection. Patients with CO-HCA-BUTI were older than those with HA-BUTI [77.0 (IQR: 69.0-83.0) vs. 71.0 (IQR: 61.5-78.5); $p<0.001]$ and had more presence of underlying diseases [209/223 $(93.72 \%)$ vs. $193 / 220$ (87.72\%); $p=0.029]$, although no differences were observed in Charslon index $(p=0.94)$.
In both groups, approximately $70 \%$ of the patients had received prior antibiotics; the most frequently used in both group included: nonantipseudomonal penicillins and cephalosporins (mainly amoxicillin, amoxicillin-clavulanate, ceftriaxone, cefixime and cefuroxime). About $20 \%$ of patients had received carbapenems. Fluoroquinolones were more frequently used in CO-HCA-BUTI patients than in the HA group $[46 / 223,20.62 \%$ vs. $26 / 220,11.81 \%$; $p<0.012]$. Clinical data and outcomes are shown in Table 2. Pitt score was higher in the CO-HCA-BUTI group ( $p=0.026)$. There were no differences between groups in either the rate of appropriate empirical treatment or duration of antibiotic therapy.

\section{Microbiological Studies}

Microbiological data are presented in Table 3. Overall, 443 episodes were included; 22 of them $(4.97 \%)$ were polymicrobial bacteremia (3 of them with 3 strains) with a total of 468 isolates. The causative microorganisms isolated were: E. coli 234/468 (50.0\%), Klebsiella pneumoniae 99/468 (21.2\%), Proteus spp. 21/468 (4.5\%), other Enterobacterales 51/468 (10.9\%), P. aeruginosa 41/468 (8.8\%) and Enterococcus spp. 22/468 (4.7\%): E. faecalis $15 / 22,(68.2 \%)$ and E. faecium $7 / 22(31.8 \%)$.

A very high percentage of pathogens with an MDR profile was observed in both groups. Although MDR rates were slightly higher in COHCA-BUTI, differences did not reach statistical significance $[146 / 223(65.47 \%)$ CO-HCA-BUTI vs. 125/220 (56.81\%) HA-BUTI; $p=0.11$ ].

Regarding Enterobacterales, among MDR strains, six had XDR profile $(4 K$. pneumoniae and 2 E. cloacae) and one PDR pattern ( $K$. pneumoniae). ESBL-producing isolates were identified in 117/405 (28.89\%) isolates: 73 E. coli, 39 K. pneumoniae, 1 Proteus spp. and 4 in other Enterobacterales. We also identified 12 carbapenemases ( 1 E. coli, $6 \mathrm{~K}$. pneumoniae, $1 \mathrm{~K}$. oxytoca, 3 E. cloacae and $1 \mathrm{M}$. morganii). No differences were observed in the rates of ESBL or carbapenemases-producing isolates when comparing groups. Overall, the more active antibiotics among Enterobacterales isolates were: 
Table 1 Baseline characteristics of patients with bacteremic urinary tract infections and comparative analysis according to the site of acquisition of the infection

\begin{tabular}{|c|c|c|c|c|}
\hline Variable & $\begin{array}{l}\text { All cases } \\
(n=443)\end{array}$ & $\begin{array}{l}\text { Community-onset healthcare- } \\
\text { associated BUTI }(n=223)\end{array}$ & $\begin{array}{l}\text { Hospital-acquired } \\
\text { BUTI }(n=220)\end{array}$ & $p$ \\
\hline \multicolumn{5}{|l|}{ Baseline features } \\
\hline Gender (male) & $288(65.0)$ & $145(65.0)$ & $143(65.0)$ & 1.00 \\
\hline Age, years, median (IQR) & $\begin{array}{l}74.0 \\
\quad(65.0-82.0)\end{array}$ & $77.0(69.0-83.0)$ & $71.0(61.5-78.5)$ & $<0.001$ \\
\hline Charlson index, median (IQR) & $3(2-5)$ & $3(2-6)$ & $3(2-5)$ & 0.944 \\
\hline McCabe score II-III & $157(35.5)$ & $76(34.1)$ & $81(36.99)$ & 0.552 \\
\hline Any underlying disease & $402(90.7)$ & $209(93.7)$ & $193(87.7)$ & 0.029 \\
\hline Diabetes Mellitus & $128(28.9)$ & $65(29.1)$ & $63(28.6)$ & 0.91 \\
\hline Chronic renal failure & $135(30.5)$ & $61(27.4)$ & $74(33.6)$ & 0.15 \\
\hline Chronic pulmonary disease & $59(13.3)$ & $32(14.3)$ & $27(12.3)$ & 0.52 \\
\hline Cardiovascular disease & $135(30.5)$ & $60(26.9)$ & $75(34.1)$ & 0.10 \\
\hline Chronic liver disease & $26(5.9)$ & $9(4.0)$ & $17(7.7)$ & 0.098 \\
\hline $\begin{array}{l}\text { Vascular/degenerative brain } \\
\text { disease }\end{array}$ & $94(21.2)$ & $58(26.0)$ & $36(16.4)$ & 0.013 \\
\hline Malignant disease & $182(41.1)$ & $104(46.6)$ & $78(35.5)$ & 0.017 \\
\hline Immunosuppressive therapy & $121(27.4)$ & $50(22.4)$ & $71(32.4)$ & 0.018 \\
\hline \multicolumn{5}{|l|}{ Previous urological history } \\
\hline $\begin{array}{l}\text { Recurrent UTI ( }>2 \text { episodes/ } \\
\text { year) }\end{array}$ & $84(19.6)$ & $64(29.8)$ & $20(9.4)$ & $<0.001$ \\
\hline $\begin{array}{l}\text { Structural urinary tract } \\
\text { abnormalities }\end{array}$ & $141(32.1)$ & $84(38.0)$ & $57(26.1)$ & 0.008 \\
\hline Indwelling urinary devices & $269(60.7)$ & $120(53.8)$ & $149(67.7)$ & 0.003 \\
\hline Urological malignancy & $111(25.1)$ & $74(33.2)$ & $37(16.8)$ & $<0.001$ \\
\hline
\end{tabular}

Friedman criteria

Previous hospitalization

$146(65.5)$

(90 days)

Indwelling urinary devices ${ }^{a}$

$112(50.2)$

Residing in long-term care

$50(22.4)$

facility

Previous ev. chemotherapy

$20(9.0)$

(30 days)

Previous ev. therapy in day

$10(4.5)$

hospital (30 days) 
Table 1 continued

\begin{tabular}{|c|c|c|c|c|}
\hline Variable & $\begin{array}{l}\text { All cases } \\
(n=443)\end{array}$ & $\begin{array}{l}\text { Community-onset healthcare- } \\
\text { associated BUTI }(n=223)\end{array}$ & $\begin{array}{l}\text { Hospital-acquired } \\
\text { BUTI }(n=220)\end{array}$ & $p$ \\
\hline Hemodialysis program & & $4(1.8)$ & & \\
\hline $\begin{array}{l}\text { Specialized ambulatory nursing } \\
\text { care ( } 30 \text { days) }\end{array}$ & & $10(4.5)$ & & \\
\hline \multicolumn{5}{|l|}{ Prior antimicrobial therapy } \\
\hline Any antibiotic (90 days) & $315(71.1)$ & $163(73.1)$ & $152(69.1)$ & 0.353 \\
\hline Fluoroquinolones & $72(16.3)$ & $46(20.6)$ & $26(11.8)$ & 0.012 \\
\hline Trimethoprim/sulfamethoxazole & $35(7.9)$ & $13(5.8)$ & $22(10.0)$ & 0.104 \\
\hline Fosfomycin & $59(13.3)$ & $26(11.7)$ & $33(15)$ & 0.30 \\
\hline $\begin{array}{l}\text { Non antipseudomonal } \\
\text { penicillins }\end{array}$ & $130(29.3)$ & $63(28.3)$ & $67(30.5)$ & 0.61 \\
\hline Antipseudomonal penicillins & $58(13.1)$ & $20(9.0)$ & $38(17.3)$ & 0.010 \\
\hline $\begin{array}{l}\text { Non-antipseudomonal } \\
\text { cephalosporins }\end{array}$ & $121(27.3)$ & $67(30.0)$ & $54(24.5)$ & 0.194 \\
\hline $\begin{array}{l}\text { Antipseudomonal } \\
\text { cephalosporins }\end{array}$ & $64(14.5)$ & $37(16.6)$ & $27(12.3)$ & 0.196 \\
\hline Carbapenems & $87(19.6)$ & $41(18.4)$ & $46(20.9)$ & 0.50 \\
\hline Aminoglycosides & $12(2.7)$ & $8(3.6)$ & $4(1.8)$ & 0.25 \\
\hline Ceftolozane-tazobactam & $12(2.7)$ & $1(0.5)$ & $11(5)$ & 0.003 \\
\hline
\end{tabular}

Data are presented as no. (\%) unless otherwise specified BUTI, bacteremic urinary tract infection; IQR, interquartile range; UTI, urinary tract infection

a Indwelling urinary devices or had conducted an invasive urinary procedure within the last 30 days

colistin $5.96 \%$ of resistance excluding isolates of intrinsically resistant species, respectively), amikacin (5.99\% resistance) and ceftolozanetazobactam (7.20\% resistance).

Regarding $P$. aeruginosa, MDR phenotype was documented in 12/41 (29.27\%) isolates; among them, 5 (12.20\%) were XDR. Only two isolates produced MBLs. Antibiotics with lower rates of resistance for $P$. aeruginosa were: colistin (0\%), amikacin (0\%) and ceftolozane/tazobactam (5.3\%). Only the two MBL-producing isolates were resistant to ceftolozane/tazobactam. Finally, among episodes caused by enterococci $(n=22)$, all $E$. faecium strains $(n=7)$ showed an MDR phenotype.

\section{Clinical Outcomes}

\section{Mortality Analysis}

Thirty-day mortality occurred in 29 patients $(6.55 \%)$, and no differences were observed between the two groups. Unadjusted and adjusted 30-day mortality is shown in Table 4. The McCabe score was not included in the multivariable model to avoid collinearity. After adjustment, mortality was associated with the severity of the underlying disease according to the Charlson index (aHR1.19 [95\% CI, 1.04-1.37]) and with being immunosuppressed (aHR 2.24 [95\% CI, 1.00-5.00]). In the multivariable analysis, mortality was not associated 
Table 2 Clinical characteristics and outcome of patients according to the site of acquisition of the bacteremic urinary tract infection

\begin{tabular}{|c|c|c|c|c|}
\hline Variable & $\begin{array}{l}\text { All cases } \\
(n=443)\end{array}$ & $\begin{array}{l}\text { Community-onset healthcare- } \\
\text { associated BUTI }(n=223)\end{array}$ & $\begin{array}{l}\text { Hospital-acquired } \\
\text { BUTI }(n=220)\end{array}$ & $p$ \\
\hline \multicolumn{5}{|l|}{ Clinical presentation } \\
\hline Pitt score $>2$ & $160(36.2)$ & $92(41.3)$ & $68(31.1)$ & 0.026 \\
\hline Septic shock & $59(14.0)$ & $36(16.9)$ & $23(11.1)$ & 0.088 \\
\hline ICU admission required & $82(18.7)$ & $22(9.9)$ & $60(27.9)$ & $<0.001$ \\
\hline Irritative urinary symptoms & $134(31.9)$ & $86(40.4)$ & $48(23.2)$ & $<0.001$ \\
\hline Renal pain & $47(11.2)$ & $32(15)$ & $15(7.2)$ & 0.011 \\
\hline Prostate pain in DRE & $6(2.8)$ & $2(1.0)$ & $8(1.9)$ & 0.165 \\
\hline Temperature $>38^{\circ} \mathrm{C}$ & $352(83.8)$ & $186(87.3)$ & $166(80.2)$ & 0.047 \\
\hline $\begin{array}{l}\text { Leukocytes }\left(10^{3} / \mathrm{ul}\right) \text {, median } \\
\quad(\mathrm{IQR})\end{array}$ & $12(8.3-16.8)$ & $12.1(8.5-17.7)$ & $11.2(7.8-16.3)$ & 0.316 \\
\hline Polimicrobial infection & $22(4.97)$ & $14(6.3)$ & $13(5.9)$ & 0.364 \\
\hline MDR profile & $271(61.2)$ & $146(65.5)$ & $125(56.8)$ & 0.062 \\
\hline \multicolumn{5}{|l|}{ Treatment } \\
\hline $\begin{array}{l}\text { Appropriate empirical } \\
\text { treatment }\end{array}$ & $359(81.0)$ & $183(82.1)$ & $176(80.0)$ & 0.580 \\
\hline Fluoroquinolones & $134(30.3)$ & $61(27.3)$ & $73(33.2)$ & 0.182 \\
\hline Ampicillin & $39(8.8)$ & $25(11.2)$ & $14(6.4)$ & 0.072 \\
\hline Amoxicillin-clavulanate & $98(22.1)$ & $55(25.0)$ & $43(19.3)$ & 0.147 \\
\hline $\begin{array}{l}\text { Non-antipseudomonal } \\
\text { cephalosporins }\end{array}$ & $159(35.9)$ & $102(45.7)$ & $57(25.9)$ & $<0.001$ \\
\hline $\begin{array}{l}\text { Antipseudomonal } \\
\text { cephalosporins }\end{array}$ & $61(13.8)$ & $35(15.7)$ & $26(11.8)$ & 0.236 \\
\hline Piperacillin-tazobactam & $108(24.4)$ & $49(22.0)$ & $59(26.8)$ & 0.235 \\
\hline Ertapenem & $116(26.2)$ & $67(30.0)$ & $49(22.3)$ & 0.063 \\
\hline Imipenem & $40(9.0)$ & $23(10.3)$ & $17(7.7)$ & 0.342 \\
\hline Meropenem & $157(35.44)$ & $68(30.5)$ & $89(40.5)$ & 0.028 \\
\hline Aztreonam & $9(2.0)$ & $3(1.4)$ & $6(2.8)$ & 0.303 \\
\hline Aminoglycosides & $53(12.0)$ & $27(12.1)$ & $26(11.8)$ & 0.925 \\
\hline Vancomycin & $12(2.7)$ & $4(1.79)$ & $8(3.6)$ & 0.232 \\
\hline Ceftolozane-tazobactam & $3(0.67)$ & $0(0)$ & $3(1.4)$ & 0.080 \\
\hline $\begin{array}{l}\text { Antibiotic treatment duration } \\
\quad(\text { days }), \text { mean } \pm S D\end{array}$ & $15.43 \pm 6.57$ & $15.08 \pm 5.67$ & $15.78 \pm 7.18$ & 0.255 \\
\hline
\end{tabular}


Table 2 continued

\begin{tabular}{|c|c|c|c|c|}
\hline Variable & $\begin{array}{l}\text { All cases } \\
(n=443)\end{array}$ & $\begin{array}{l}\text { Community-onset healthcare- } \\
\text { associated BUTI }(n=223)\end{array}$ & $\begin{array}{l}\text { Hospital-acquired } \\
\text { BUTI }(n=220)\end{array}$ & $p$ \\
\hline $\begin{array}{l}\text { Urological intervention } \\
\text { required }\end{array}$ & $116(26.2)$ & $60(26.9)$ & $56(25.6)$ & 0.750 \\
\hline \multicolumn{5}{|l|}{ Outcome } \\
\hline \multicolumn{5}{|l|}{ Clinical assessment at $48-72 \mathrm{~h}$} \\
\hline Afebrile & $393(89.5)$ & $193(87.3)$ & $200(91.7)$ & 0.131 \\
\hline $\begin{array}{l}\text { Persistence of sepsis signs/ } \\
\text { symptoms }\end{array}$ & $49(11.2)$ & & & 0.686 \\
\hline Clinical cure & $263(59.4)$ & $119(53.4)$ & $144(65.5)$ & 0.010 \\
\hline $\begin{array}{l}\text { Median hospital stay since } \\
\text { BUTI, d (IQR) }\end{array}$ & $12(8-20)$ & $10(7-14)$ & $16(10-29.5)$ & $<0.001$ \\
\hline Mortality at day 30 & $32(7.2)$ & $17(7.6)$ & $15(6.8)$ & 0.743 \\
\hline Mortality related with infection & $9(2)$ & $6(2.7)$ & $3(1.4)$ & 0.322 \\
\hline
\end{tabular}

Data are presented as no. (\%) unless otherwise specified

IQR, interquatile range; SD, standard deviation. BUTI, bacteremic urinary tract infection; ICU, intensive care unit; irritative urinary symptoms include dysuria, frequency, gross hematuria and urgency; DRE, digital rectal examination; urological intervention required included endoscopic, percutaneous or surgical drainage; MDR, multidrug resistant

with the site of acquisition or the presence of MDR profile.

\section{Length of Stay and Clinical Cure}

The median hospital length of stay after the onset of bacteremia was higher in those patients with HA-BUTI $(p>0.001)$. No significant differences were observed in the rate of clinical stability reached at $48-72 \mathrm{~h}$ when comparing CO-HCA-BUTI and HA-BUTI [177/223 (79.37\%) CO-HCA-BUTI vs. 181/220 (82.23\%) HA-BUTI; $p=0.42]$. In multivariable logistic regression analysis (Table 5) of clinical cure at hospital discharge, both CO-HCA-BUTI (aOR 0.61 [95\% $\mathrm{CI}, 0.40-0.93])$ and urinary tract abnormalities (aOR 0.56 [95\% CI, 0.36-0.88]) were associated with lower odds of achieving clinical cure at hospital discharge. MDR profile was not associated with a worse clinical cure.

\section{Empirical Antibiotic Treatment}

Table S1 in Supplementary Material summarizes the parameters related to receiving inadequate empirical antibiotic treatment. Overall, 85 patients $(19.18 \%)$ received inadequate initial therapy. Among them, the most frequently used antibiotics were: non-antipseudomonal cephalosporins (26 patients), penicillins plus $\beta$ lactamase inhibitors (18 patients), antipseudomonal penicillins plus $\beta$-lactamase inhibitors (13 patients) and fluoroquinolones (13 patients). The median time until receiving an active therapy was 3 days. No differences in length of stay were observed between patients who received adequate and inadequate empirical treatment, although the median hospital stay after the onset of BUTI (days) was slightly longer in those who received inadequate empirical treatment [11 (IQR 8-20) vs. 13 $(8-22) ; p=0.43]$. In a multivariable analysis, MDR profile (aOR 3.35 [1.77-6.35]), P. aeruginosa (OR 2.86 [1.27-6.44]) and Charlson index 
Table 3 Microorganisms isolated from blood cultures of patients with bacteremic urinary tract infection: antimicrobial susceptibility, resistance profile and resistance mechanisms

\begin{tabular}{|c|c|c|c|c|c|}
\hline $\begin{array}{l}\text { Uropathogen } \\
\text { isolated } \\
N \text { (\% global) }\end{array}$ & $\begin{array}{l}\text { Antibiotic resistance rate } \\
(n, \%)\end{array}$ & $\begin{array}{l}\text { All cases, } \\
n=446 \\
\text { Antibiotic } \\
\text { resistance } \\
(n, \%)\end{array}$ & $\begin{array}{l}\text { Community-onset } \\
\text { healthcare associated BUTI } \\
(n=226)\end{array}$ & $\begin{array}{l}\text { Hospital- } \\
\text { acquired BUTI } \\
(n=220)\end{array}$ & $p$ \\
\hline \multirow[t]{16}{*}{ Escherichia coli } & & 234 & 132 & 102 & \\
\hline & Penicillins & $191(81.6)$ & $110(83.3)$ & $81(79.4)$ & 0.50 \\
\hline & $\begin{array}{l}\text { Penicillins }+\beta \text {-lactamase } \\
\text { inhibitor }\end{array}$ & $82(35.0)$ & $45(34.1)$ & $37(36.3)$ & 0.78 \\
\hline & $\begin{array}{l}\text { Antipseudomonal } \\
\text { penicillins }+\beta- \\
\text { lactamase inhibitor }\end{array}$ & $52(22.2)$ & $31(23.5)$ & $21(20.6)$ & 0.6 \\
\hline & $\begin{array}{l}\text { Non antipseudomonal } \\
\text { cephalosporins }\end{array}$ & $76(32.5)$ & $47(35.6)$ & $29(28.4)$ & 0.32 \\
\hline & $\begin{array}{l}\text { Antipseudomonal } \\
\text { cephalosporins }\end{array}$ & $73(31.2)$ & $46(34.8)$ & $27(26.5)$ & 0.20 \\
\hline & Ceftolozane/tazobactam & 0 & 0 & 0 & - \\
\hline & $\begin{array}{l}\text { Monobactams } \\
\text { (Aztreonam) }\end{array}$ & $69(29.5)$ & $42(31.8)$ & $27(26.5)$ & 0.38 \\
\hline & Group 1 Carbapenems & $1(0.4)$ & 0 & $1(1)$ & 0.44 \\
\hline & Group 2 Carbapenems & 0 & 0 & 0 & - \\
\hline & Fluoroquinolones & $129(55.1)$ & $81(61.4)$ & $48(47.1)$ & 0.034 \\
\hline & Aminoglycosides & $67(28.6)$ & $13(35.6)$ & $20(19.6)$ & 0.009 \\
\hline & $\begin{array}{l}\text { Trimethoprim- } \\
\text { sulfamethoxazole }\end{array}$ & $101(43.2)$ & $55(41.7)$ & $46(45.1)$ & 0.69 \\
\hline & Fosfomycin & $83(35.5)$ & $50(37.9)$ & $33(32.4)$ & 0.41 \\
\hline & Polymyxins (Colistin) & $1(0.4)$ & $1(0.8)$ & 0 & 1.00 \\
\hline & $\operatorname{MDR}$ profile $(n, \% \mathrm{MDR})$ & $172(73.5)$ & $104(78.8)$ & $68(66.7)$ & 0.052 \\
\hline \multirow{4}{*}{$\begin{array}{l}\text { Klebsiella } \\
\text { pneumoniae }\end{array}$} & & 99 & 37 & 62 & \\
\hline & $\begin{array}{l}\text { Penicillins }+\beta \text {-lactamase } \\
\text { inhibitor }\end{array}$ & $61(61.6)$ & $19(51.4 \%)$ & $42(67.7)$ & 0.14 \\
\hline & $\begin{array}{l}\text { Antipseudomonal } \\
\text { penicillins }+\beta- \\
\text { lactamase inhibitor }\end{array}$ & $36(36.4)$ & $13(35.1)$ & $23(37.1)$ & 1.00 \\
\hline & $\begin{array}{l}\text { Non antipseudomonal } \\
\text { cephalosporins }\end{array}$ & $41(41.8)$ & $14(37.8)$ & $27(44.3)$ & 0.67 \\
\hline
\end{tabular}


Table 3 continued

\begin{tabular}{|c|c|c|c|c|c|}
\hline $\begin{array}{l}\text { Uropathogen } \\
\text { isolated } \\
N \text { (\% global) }\end{array}$ & $\begin{array}{l}\text { Antibiotic resistance rate } \\
(n, \%)\end{array}$ & $\begin{array}{l}\text { All cases, } \\
n=446 \\
\text { Antibiotic } \\
\text { resistance } \\
(n, \%)\end{array}$ & $\begin{array}{l}\text { Community-onset } \\
\text { healthcare associated BUTI } \\
(n=226)\end{array}$ & $\begin{array}{l}\text { Hospital- } \\
\text { acquired BUTI } \\
(n=220)\end{array}$ & $p$ \\
\hline & $\begin{array}{l}\text { Antipseudomonal } \\
\text { cephalosporins }\end{array}$ & $42(42.4)$ & $14(37.8)$ & $28(45.2)$ & 0.53 \\
\hline & Ceftolozane/tazobactam & $17(17.7)$ & $4(10.8)$ & $13(22.0)$ & 0.18 \\
\hline & Monobactam (Aztreonam) & $41(42.3)$ & $14(37.8)$ & $27(45.0)$ & 0.53 \\
\hline & Group 1 Carbapenems & $9(9.1)$ & $2(5.4)$ & $7(11.3)$ & 0.48 \\
\hline & Group 2 Carbapenems & $3(3.0)$ & $0(0.0)$ & $3(4.8)$ & 0.29 \\
\hline & Fluoroquinolones & $47(47.5)$ & $15(40.5)$ & $32(51.6)$ & 0.31 \\
\hline & Aminoglycosides & $39(39.4)$ & $14(37.8)$ & $25(40.3)$ & 0.84 \\
\hline & $\begin{array}{l}\text { Trimethoprim- } \\
\text { sulphamethoxazole }\end{array}$ & $44(44.4)$ & $16(43.2)$ & $28(45.2)$ & 1.00 \\
\hline & Fosfomycin & $84(87.5)$ & $33(89.2)$ & $51(86.4)$ & 0.76 \\
\hline & Polymyxins (Colistin) & $5(5.2)$ & $1(2.7)$ & $4(6.8)$ & 0.65 \\
\hline & $\operatorname{MDR}$ profile $(n, \%$ MDR $)$ & $56(56.6)$ & $19(51.4)$ & $37(59.7)$ & 0.53 \\
\hline \multirow[t]{9}{*}{ Proteus spp. } & & 21 & 14 & 7 & \\
\hline & $\begin{array}{l}\text { Penicillins }+\beta \text {-lactamase } \\
\text { inhibitor }\end{array}$ & $9(42.9)$ & $9(64.3)$ & 0 & 0.007 \\
\hline & $\begin{array}{l}\text { Antipseudomonal } \\
\text { penicillins }+\beta \text { - } \\
\text { lactamase inhibitor }\end{array}$ & 0 & 0 & 0 & - \\
\hline & $\begin{array}{l}\text { Non antipseudomonal } \\
\text { cephalosporins }\end{array}$ & $1(4.8)$ & 0 & $1(14.3)$ & 1.00 \\
\hline & $\begin{array}{l}\text { Antipseudomonal } \\
\text { cephalosporins }\end{array}$ & $1(4.8)$ & 0 & $1(14.3)$ & 1.00 \\
\hline & $\begin{array}{l}\text { Monobactams } \\
\text { (Aztreonam) }\end{array}$ & $1(4.8)$ & 0 & $1(14.3)$ & 1.00 \\
\hline & $\begin{array}{l}\text { Group } 1 \text { and group } 2 \\
\text { carbapenems }\end{array}$ & 0 & 0 & 0 & - \\
\hline & Fluoroquinolones & $11(52.4)$ & $10(71.4)$ & $1(14.3)$ & 0.02 \\
\hline & Aminoglycosides & $10(47.6)$ & $7(50.0)$ & $3(42.9)$ & 1.00 \\
\hline
\end{tabular}


Table 3 continued

\begin{tabular}{|c|c|c|c|c|c|}
\hline $\begin{array}{l}\text { Uropathogen } \\
\text { isolated } \\
N \text { (\% global) }\end{array}$ & $\begin{array}{l}\text { Antibiotic resistance } \\
\text { rate }(n, \%)\end{array}$ & $\begin{array}{l}\text { All cases, } \\
n=446 \\
\text { Antibiotic } \\
\text { resistance } \\
(n, \%)\end{array}$ & $\begin{array}{l}\text { Community-onset } \\
\text { healthcare associated BUTI } \\
(n=226)\end{array}$ & $\begin{array}{l}\text { Hospital- } \\
\text { acquired BUTI } \\
(n=220)\end{array}$ & $p$ \\
\hline & $\begin{array}{l}\text { Trimethoprim- } \\
\text { sulfamethoxazole }\end{array}$ & & & & \\
\hline & Fosfomycin & $9(42.9)$ & $7(50.0)$ & $2(28.6)$ & 0.64 \\
\hline & MDR profile $(n, \% \mathrm{MDR})$ & $11(52.4)$ & $10(71.4)$ & $1(14.3)$ & 0.02 \\
\hline \multirow{15}{*}{$\begin{array}{l}\text { Other } \\
\text { Enterobacterales }\end{array}$} & & 51 & 21 & 30 & \\
\hline & & & & & \\
\hline & $\begin{array}{l}\text { Antipseudomonal } \\
\text { penicillins }+\beta- \\
\text { lactamase inhibitor }\end{array}$ & $13(25.5)$ & $4(19.0)$ & $9(30.0)$ & 0.52 \\
\hline & $\begin{array}{l}\text { Non antipseudomonal } \\
\text { cephalosporins }\end{array}$ & $15(29.4)$ & $3(14.3)$ & $12(40.0)$ & 0.064 \\
\hline & $\begin{array}{l}\text { Antipseudomonal } \\
\text { cephalosporins }\end{array}$ & $12(23.5)$ & $3(14.3)$ & $9(30.0)$ & 0.32 \\
\hline & Ceftolozane/tazobactam & $11(21.6)$ & $2(9.5)$ & $9(30.0)$ & 0.098 \\
\hline & $\begin{array}{l}\text { Monobactam } \\
\quad(\text { Aztreonam })\end{array}$ & $12(23.5)$ & $3(14.3)$ & $9(30.0)$ & 0.32 \\
\hline & Group 1 Carbapenems & $8(15.7)$ & $2(9.5)$ & $6(20.0)$ & 0.445 \\
\hline & Group 2 Carbapenems & $1(2.0)$ & $0(0.0)$ & $1(3.3)$ & 1.00 \\
\hline & Fluoroquinolones & $11(21.6)$ & $7(33.3)$ & $4(13.3)$ & 0.17 \\
\hline & Aminoglycosides & $10(19.6)$ & $3(14.3)$ & $7(23.3)$ & 0.50 \\
\hline & $\begin{array}{l}\text { Trimethoprim- } \\
\text { sulphamethoxazole }\end{array}$ & $11(21.6)$ & $7(33.3)$ & $4(13.3)$ & 0.17 \\
\hline & Fosfomycin & $41(80.4)$ & $18(85.7)$ & $23(76.7)$ & 0.50 \\
\hline & Polymyxins (Colistin) & $16(31.4)$ & $5(23.8)$ & $11(36.7)$ & 0.375 \\
\hline & MDR profile $(n, \%$ MDR $)$ & $18(35.3)$ & $7(33.3)$ & $11(36.7)$ & 1.00 \\
\hline \multirow{3}{*}{$\begin{array}{l}\text { Pseudomonas } \\
\text { aeruginosa }\end{array}$} & & 41 & 22 & 19 & \\
\hline & $\begin{array}{l}\text { Antipseudomonal } \\
\text { penicillins }+\beta \text { - } \\
\text { lactamase inhibitor }\end{array}$ & $14(34.14)$ & $8(36.4)$ & $6(31.6)$ & 1.00 \\
\hline & $\begin{array}{c}\text { Antipseudomonal } \\
\text { cephalosporins }\end{array}$ & $13(31.7)$ & $7(31.8)$ & $6(31.6)$ & 1.00 \\
\hline
\end{tabular}


Table 3 continued

\begin{tabular}{|c|c|c|c|c|c|}
\hline \multirow[t]{8}{*}{$\begin{array}{l}\text { Uropathogen } \\
\text { isolated } \\
N \text { (\% global) }\end{array}$} & $\begin{array}{l}\text { Antibiotic resistance } \\
\text { rate }(n, \%)\end{array}$ & $\begin{array}{l}\text { All cases, } \\
n=446 \\
\text { Antibiotic } \\
\text { resistance ( } n, \\
\%)\end{array}$ & $\begin{array}{l}\text { Community-onset healthcare } \\
\text { associated BUTI }(n=226)\end{array}$ & $\begin{array}{l}\text { Hospital-acquired } \\
\text { BUTI }(n=220)\end{array}$ & $p$ \\
\hline & Ceftolozane/tazobactam & $2(4.9)$ & $1(4.5)$ & $1(5.2)$ & 1.00 \\
\hline & $\begin{array}{l}\text { Monobactams } \\
\text { (Aztreonam) }\end{array}$ & $4(9.8)$ & $2(9.1)$ & $2(10.5)$ & 1.00 \\
\hline & Group 2 Carbapenems & $17(41.5)$ & $7(31.8)$ & $10(52.6)$ & 0.22 \\
\hline & Fluoroquinolones & $15(36.6)$ & $7(31.8)$ & $8(42.1)$ & 0.53 \\
\hline & Aminoglycosides & $7(17.1)$ & $3(13.6)$ & $4(21.1)$ & 0.69 \\
\hline & Polymyxins (Colistin) & $0(0)$ & $0(0)$ & $0(0)$ & - \\
\hline & $\begin{array}{l}\text { MDR profile }(n, \% \\
\text { MDR })\end{array}$ & $12(29.3)$ & $6(27.3)$ & $6(31.6)$ & 1.00 \\
\hline
\end{tabular}

Data are presented as no. (\%)

Antimicrobial categories (antimicrobial agents tested and included): penicillins (ampicillin), penicillins $+\beta$-lactamase inhibitor (amoxicillin-clavunalate), antipseudomonal penicillins $+\beta$-lactamase inhibitor (piperacillin-tazobactam), nonantipseudomonal cephalosporines (cefotaxime), antipseudomonal cephalosporines (ceftazidime, cefepime), group 1 carbapenems (ertapenem), group 2 carbapenems (imipenem, meropenem), monobactam (aztreonam) fluoroquinolones (ciprofloxacin), aminoglycosides (gentamicin, tobramycin, amikacin), polymyxins (colistin). Proteus spp. included: 19 P. mirabilis, 1 P. vulgaris and 1 Proteus spp. Other Enterobacterales included: 15 Enterobacter cloacae complex, $9 \mathrm{~K}$. aerogenes, 7 K. oxytoca, 8 Serratia marcescens, 4 Morganella morganii, 1 Providencia stuartii, 3 Citrobacter koseri, 2 C. freundii and 1 C. amalonatycus. MDR, multidrug resistance profile was defined as a strain non-susceptible to at least one agent in $\geq 3$ antimicrobial categories

BUTI, bacteremic urinary tract infection; MDR, multidrug-resistant

(aOR 1.11 [1.01-1.23]) were the independent predictors of receiving inadequate empirical treatment, whereas the initial presentation with septic shock was a protective factor (aOR 0.38 [0.15-0.96]).

\section{DISCUSSION}

Healthcare-associated infections represent a current major health problem [2]. Demographic changes, aging and a higher grade of complexity of medical treatments are among the reasons for the increasing number of HCA infections during recent years $[7,16]$. Furthermore, many patients with multiple underlying diseases and/ or invasive devices, who were traditionally treated in acute care hospitals, are currently managed in primary care centers, in long-term care facilities or even at home [33]. Previous studies have shown that CO-HCA infections represent a different entity from other community-acquired infections $[17,18]$. However, few studies have aimed to clarify whether COHCA infections should be managed as HA infections [18, 34, 35]. We carried out a multicenter prospective study to gain a better understanding of CO-HCA and HA infections. Due to the huge clinical and economic burden of UTI on the healthcare systems [3, 8], and to avoid bias derived from including different sources of infection, we focused our study on BUTI.

In our study, patients with CO-HCA infections were older and had more underlying 
Table 4 Univariate and multivariable analysis of parameters predicting overall mortality in bacteremic urinary tract infection

\begin{tabular}{|c|c|c|c|c|c|c|}
\hline & \multicolumn{6}{|c|}{ Overall mortality $(n=443,30$-day mortality $=29)$} \\
\hline & $\begin{array}{l}\text { Deceased } \\
(n=29)\end{array}$ & $\begin{array}{l}\text { Alive } \\
(n=414)\end{array}$ & $\begin{array}{l}\text { Unadjusted HR } \\
(95 \% \mathrm{CI})\end{array}$ & $p$ & $\begin{array}{l}\text { Adjusted HR } \\
(95 \% \mathrm{CI})\end{array}$ & $p$ \\
\hline Gender (male) & $21(72.41)$ & $267(64.49)$ & $1.43(0.63-3.23)$ & 0.39 & & \\
\hline Age, years, median (IQR) & $79(66-84)$ & $74(65-82)$ & $1.02(0.99-1.05)$ & 0.27 & $1.02(0.99-1.06)$ & 0.23 \\
\hline Charlson index, median (IQR) & $6(2-7)$ & $3(2-5)$ & $1.24(1.09-1.40)$ & 0.001 & $1.19(1.04-1.37)$ & 0.012 \\
\hline McCabe score II-III & $26(89.66)$ & $131(31.64)$ & $16.89(5.11-55.80)$ & $<0.001$ & - & - \\
\hline Diabetes mellitus & $8(27.59)$ & $120(28.99)$ & $0.94(0.42-2.12)$ & 0.88 & & \\
\hline Chronic renal failure & $11(37.93)$ & $124(29.95)$ & $1.44(0.68-3.05)$ & 0.34 & & \\
\hline Chronic pulmonary disease & $4(13.79)$ & $55(132.85)$ & $1.06(0.37-3.04)$ & 0.92 & & \\
\hline Cardiovascular disease & $10(34.48)$ & $125(30.19)$ & $1.23(0.57-2.64)$ & 0.60 & & \\
\hline Chronic liver disease & $3(10.34)$ & $23(5.56)$ & $1.86(0.56-6.16)$ & 0.31 & & \\
\hline $\begin{array}{l}\text { Vascular/degenerative brain } \\
\text { disease }\end{array}$ & $8(27.59)$ & $86(20.77)$ & $1.45(0.64-3.26)$ & 0.38 & & \\
\hline Malignant disease & $15(51.72)$ & $167(40.34)$ & $1.54(0.74-3.20)$ & 0.24 & & \\
\hline Immunosuppressive therapy & $13(44.83)$ & $108(26.09)$ & $2.18(1.05-4.53)$ & 0.037 & $2.23(1.00-5.00)$ & 0.05 \\
\hline $\begin{array}{l}\text { Recurrent UTI ( }>2 \text { episodes/ } \\
\text { year) }\end{array}$ & $7(24.14)$ & $77(18.60)$ & $1.53(0.64-3.65)$ & 0.33 & & \\
\hline $\begin{array}{l}\text { Structural urinary tract } \\
\text { abnormalities }\end{array}$ & $15(51.72)$ & $126(30.43)$ & $2.53(1.20-5.31)$ & 0.014 & $2.11(0.98-4.55)$ & 0.06 \\
\hline Indwelling urinary devices & $20(68.97)$ & $249((60.14)$ & $1.46(0.66-3.20)$ & 0.35 & & \\
\hline Site of infection (CO-HCA) & $16(55.17)$ & $207(50.00)$ & $1.23(0.59-2.55)$ & 0.58 & $1.01(0.46-2.19)$ & 0.99 \\
\hline $\begin{array}{l}\text { Previous antibiotic use } \\
\quad(3 \text { months })\end{array}$ & $24(82.76)$ & $291(70.29)$ & $1.98(0.76-5.20)$ & 0.16 & & \\
\hline Pitt score $>2$ & $12(41.38)$ & $148(35.75)$ & $1.25(0.60-2.62)$ & 0.55 & & \\
\hline Septic shock & $5(17.24)$ & $54(13.04)$ & $1.48(0.56-3.93)$ & 0.43 & & \\
\hline ICU admission required & $5(17.24)$ & $77(18.60)$ & $0.90(0.34-2.36)$ & 0.83 & & \\
\hline Polimicrobial infection & $1(3.45)$ & $21(5.07)$ & $0.68(0.09-4.98)$ & 0.70 & & \\
\hline Resistance profile (MDR) & $22(75.86)$ & $249(60.14)$ & $2.04(0.87-4.78)$ & 0.10 & $1.80(0.75-4.33)$ & 0.19 \\
\hline Appropriate empirical treatment & $18(62.07)$ & $340(82.13)$ & $0.37(0.17-0.78)$ & 0.009 & $0.61(0.27-1.38)$ & 0.23 \\
\hline $\begin{array}{l}\text { Persistence of sepsis signs/ } \\
\text { symptoms at } 48-72 \mathrm{~h}\end{array}$ & $5(17.24)$ & $44(10.63)$ & $1.90(0.72-5.02)$ & 0.20 & & \\
\hline Escherichia coli & $16(55.17)$ & $214(51.69)$ & $1.15(0.55-2.39)$ & 0.71 & & \\
\hline Klebsiella pneumoniae & $5(17.24)$ & $94(22.70)$ & $0.72(0.28-1.89)$ & 0.51 & & \\
\hline
\end{tabular}


Table 4 continued

\begin{tabular}{|c|c|c|c|c|c|c|}
\hline & \multicolumn{6}{|c|}{ Overall mortality $(n=443,30$-day mortality $=29)$} \\
\hline & $\begin{array}{l}\text { Deceased } \\
(n=29)\end{array}$ & $\begin{array}{l}\text { Alive } \\
(n=414)\end{array}$ & $\begin{array}{l}\text { Unadjusted HR } \\
(95 \% \mathrm{CI})\end{array}$ & $p$ & $\begin{array}{l}\text { Adjusted HR } \\
\text { (95\% CI) }\end{array}$ & $p$ \\
\hline Proteus spp. & $2(6.90)$ & $19(4.59)$ & $1.56(0.37-6.54)$ & 0.55 & & \\
\hline Other Enterobacterales & $2(6.90)$ & $49(11.84)$ & $0.57(0.13-2.38)$ & 0.44 & & \\
\hline Pseudomonas aeruginosa & $4(13.79)$ & $37(8.94)$ & $1.55(0.54-4.44)$ & 0.83 & & \\
\hline Enterococcus spp. & $2(6.90)$ & $20(4.83)$ & $1.41(0.33-5.91)$ & 0.64 & & \\
\hline
\end{tabular}

diseases than those with HA-BUTI. The MDR rate was high, but similar between the two groups. Several studies have also shown that CO-HCA infections occur in vulnerable populations $[13,14,17,18,35]$. The rising rates of elderly and complex patients managed in the community setting could explain this fact. In line with our results, previous studies have found high rates of antibiotic resistance in COHCA infections [17, 18, 33]. Therefore, although CO-HCA infections were initially considered an intermediate entity between community-acquired and HA infections [35], it seems they are more prone to HA infections, at least in terms of patients' characteristics and microbiology $[17,18]$. Although no differences were found in mortality rates, patients with HA-BUTI had a better clinical cure than those with CO-HCA infection. As UTIs are usually associated with a low mortality rate [29], clinical cure could be a more accurate outcome to assess BUTI prognosis.

Worryingly, our study shows a high rate of healthcare-related patients with previous use of antimicrobial agents. In fact, the European Centre for Disease Prevention and Control data demonstrated high antibiotic consumption rates in Spain, being the fifth country in Europe in consumption of systemic antimicrobials in the community and hospital sector in 2019 [36]. Several studies have demonstrated that previous antibiotic exposure is a strong risk factor for colonization and infection with a drug-resistant pathogen [37, 38]. The high percentages of prior antibiotic may explain, at least in part, the high rates of MDR infections observed in our study. In this scenario, Antimicrobial Stewardship programs are essential to enhance appropriate use of antimicrobial therapy.

Of relevance is the dramatic increase of multidrug resistance rates compared with previous studies carried out in our country $[16,18]$. In recent years MDR Enterobacterales, mainly ESBL producers, have emerged as important causes of community-onset pathogens worldwide [39]. In this context, developing public health strategies to control and reduce the spread of MDR strains in healthcare settings, such as long-term care facilities, is essential [40].

In our study, receiving inadequate empirical antibiotic treatment was independently associated with MDR profile and with $P$. aeruginosa. Doubtlessly, one of the main consequences of multidrug resistance is that patients are at higher risk of receiving inadequate empirical antibiotic treatment. The delay in receiving effective antibiotics has been linked to worse outcomes, including mortality $[29,30]$. However, inadequate empirical treatment was not associated with worse outcomes in our cohort. The small number of patients who received inadequate empirical treatment might contribute to finding any statistically significant differences. On the other hand, this finding could be partly explained because patients who were less likely to receive inadequate empirical treatments were those with septic shock, in whom time is a critical factor [41]. Another possible explanation is the relatively low 
Table 5 Univariate and multivariable analysis of parameters predicting clinical cure in bacteremic urinary tract infection

\begin{tabular}{|c|c|c|c|c|c|c|}
\hline & \multicolumn{6}{|c|}{ Clinical cure $(n=443$, clinical cure $=263)$} \\
\hline & $\begin{array}{l}\text { Cure } \\
(n=263)\end{array}$ & $\begin{array}{l}\text { Failure } \\
(N=180)\end{array}$ & $\begin{array}{l}\text { Unadjusted OR } \\
(95 \% \mathrm{CI})\end{array}$ & $p$ & $\begin{array}{l}\text { Adjusted OR } \\
(95 \% \mathrm{CI})\end{array}$ & $p$ \\
\hline Gender (male) & $158(60.07)$ & $130(72.22)$ & $0.58(0.38-0.87)$ & 0.009 & $0.67(0.43-1.06)$ & 0.09 \\
\hline Age, years, median (IQR) & $75(66-82)$ & $73(64-82)$ & $1.00(0.99-1.02)$ & 0.68 & & \\
\hline Charlson index, median (IQR) & $3(2-5)$ & $3(2-6)$ & $1.00(0.93-1.07)$ & 0.94 & & \\
\hline McCabe score II-III & $96(36.50)$ & $61(33.89)$ & $1.11(0.75-1.66)$ & 0.60 & & \\
\hline Diabetes mellitus & $80(30.42)$ & $48(26.67)$ & $1.20(0.79-1.83)$ & 0.39 & & \\
\hline Chronic renal failure & $83(31.56)$ & $52(28.89)$ & $1.14(0.75-1.72)$ & 0.55 & & \\
\hline Chronic pulmonary disease & $36(13.69)$ & $23(12.78)$ & $1.08(0.62-1.90)$ & 0.78 & & \\
\hline Cardiovascular disease & $83(31.56)$ & $52(28.89)$ & $1.14(0.75-1.72)$ & 0.55 & & \\
\hline Chronic liver disease & $15(5.70)$ & $11(6.11)$ & $0.93(0.42-2.07)$ & 0.86 & & \\
\hline $\begin{array}{l}\text { Vascular/degenerative brain } \\
\text { disease }\end{array}$ & $59(22.43)$ & $35(19.44)$ & $1.20(0.75-1.92)$ & 0.45 & & \\
\hline Malignant disease & $98(37.26)$ & $84(46.67)$ & $0.68(0.46-1.00)$ & 0.049 & $0.72(0.48-1.09)$ & 0.12 \\
\hline Immunosuppressive therapy & $77(29.28)$ & $44(24.44)$ & $1.27(0.82-1.96)$ & 0.28 & & \\
\hline $\begin{array}{l}\text { Recurrent UTI ( }>2 \text { episodes/ } \\
\text { year) }\end{array}$ & $54(20.53)$ & $30(16.67)$ & $1.27(0.77-2.08)$ & 0.35 & & \\
\hline $\begin{array}{l}\text { Structural urinary tract } \\
\text { abnormalities }\end{array}$ & $66(17.36)$ & $75(41.67)$ & $0.46(0.31-0.70)$ & $<0.001$ & $0.56(0.36-0.88)$ & 0.01 \\
\hline Indwelling urinary devices & $161(61.22)$ & $108(60.00)$ & $1.05(0.71-1.55)$ & 0.80 & & \\
\hline Site of infection (CO-HCA) & $119(45.24)$ & $104(57.7)$ & $0.60(0.41-0.89)$ & 0.010 & $0.61(0.40-0.93)$ & 0.02 \\
\hline $\begin{array}{l}\text { Previous antibiotic use } \\
\quad(3 \text { months })\end{array}$ & $187(71.10)$ & $128(71.11)$ & $1.00(0.66-1.52)$ & 1.00 & & \\
\hline Pitt score $>2$ & $101(38.40)$ & $59(32.78)$ & $1.27(0.85-1.89)$ & 0.24 & & \\
\hline Septic shock & $41(15.59)$ & $18(10.00)$ & $1.62(0.90-2.93)$ & 0.11 & & \\
\hline ICU admission required & $58(22.05)$ & $24(13.33)$ & $1.78(1.06-2.99)$ & 0.03 & $1.74(0.96-3.05)$ & 0.052 \\
\hline Polimicrobial infection & $14(5.32)$ & $8(4.44)$ & $1.21(0.50-2.94)$ & 0.68 & & \\
\hline Resistance profile (MDR) & $164(62.35)$ & $107(59.44)$ & $1.13(0.77-1.67)$ & 0.53 & $1.17(0.75-1.83)$ & 0.49 \\
\hline Appropriate empirical treatment & $218(82.89)$ & $140(77.78)$ & $1.38(0.86-2.23)$ & 0.18 & $1.39(0.82-2.33)$ & 0.22 \\
\hline $\begin{array}{l}\text { Persistence of sepsis signs/ } \\
\text { symptoms at } 48-72 \mathrm{~h}\end{array}$ & $26(9.89)$ & $23(12.78)$ & $0.74(0.41-1.34)$ & 0.32 & & \\
\hline Escherichia coli & $146(55.51)$ & $84(46.67)$ & $1.43(0.97-2.09)$ & 0.07 & $1.01(0.60-1.69)$ & 0.98 \\
\hline Klebsiella pneumoniae & $51(19.39)$ & $48(26.27)$ & $0.66(0.42-1.04)$ & 0.07 & $0.59(0.33-1.05)$ & 0.08 \\
\hline Proteus spp. & $11(4.18)$ & $10(5.56)$ & $0.74(0.31-1.79)$ & 0.51 & & \\
\hline
\end{tabular}


Table 5 continued

\begin{tabular}{lclllll}
\hline & \multicolumn{2}{l}{ Clinical cure $(\boldsymbol{n}=\mathbf{4 4 3}$, clinical cure $=\mathbf{2 6 3})$} & & \\
\cline { 2 - 6 } & $\begin{array}{l}\text { Cure } \\
(\boldsymbol{n}=\mathbf{2 6 3})\end{array}$ & $\begin{array}{l}\text { Failure } \\
(\boldsymbol{N}=\mathbf{1 8 0})\end{array}$ & $\begin{array}{l}\text { Unadjusted OR } \\
(\mathbf{9 5 \%} \mathbf{C I})\end{array}$ & $\boldsymbol{p}$ & $\begin{array}{l}\text { Adjusted OR } \\
(\mathbf{9 5 \%} \text { CI })\end{array}$ & $\boldsymbol{p}$ \\
\hline Other Enterobacterales & $30(11.41)$ & $21(11.67)$ & $0.97(0.54-1.76)$ & 0.93 & \\
Pseudomonas aeruginosa & $24(9.13)$ & $17(9.44)$ & $0.96(0.50-1.85)$ & 0.91 & \\
Enterococcus spp. & $12(4.56)$ & $10(5.56)$ & $0.81(0.31-1.92)$ & 0.64 & \\
\hline
\end{tabular}

mortality rate of UTI compared with other sources of infections [29].

Some limitations of our study should be recognized. First, we did not include non-hospitalized patients, so our results cannot be extrapolated to outpatients. Second, the comparator groups may be imbalanced in terms of bacteremia duration. Although the communityonset group is likely to have a more prolonged duration before the hospital admission, in routine clinical practice, determining the exact difference in bacteremia duration between groups is very complicated. Third, the observation period for clinical cure at hospital discharge may be biased between groups as lengths of stay may differ for other reasons beyond the BUTI episode. Fourth, our results may not be generalizable to other countries with a different epidemiology. Finally, antibiotic dosing was not recorded. Antibiotic dosing is associated with appropriate treatment; thus, not collecting this information may represent a lost opportunity to evaluate the role of timely and appropriate antibiotic exposures.

\section{CONCLUSIONS}

In summary, the present study represents the largest study published to date with the aim of evaluating differences between CO-HCA-BUTI and HA-BUTI. Our study shed light on the burden of CO-HCA infections, at least regarding UTI. CO-HCA-BUTIs occur in complex patients, who are more elderly and have more underlying comorbidities. The rate of MDR infections was similar in both groups, and worryingly high overall. We did not find differences in either mortality or the probability of receiving inappropriate empirical antibiotic treatment between the two groups. However, CO-HCABUTIs were associated with a worse clinical cure. Our results suggest that CO-HCAs are similar or even worse than HA-BUTIs. These results should be considered when prescribing empirical antibiotic treatments. However, it should be noted that CO-HCA infections include a very heterogeneous group of patients and, in the face of antimicrobial stewardship strategies, an important challenge will be to differentiate which patients are at a higher risk of developing a MDR infections within this huge group.

\section{ACKNOWLEDGEMENTS}

We thank the patients who participated in the study.

Collaborating investigator list: Ana Siverio (Microbiology Service, Hospital del Mar, Institut Hospital del Mar d'Investigacions Mèdiques, Barcelona, Spain). Desiré Gijón (Microbiology Service, Hospital Universitario Ramón y CajalIRYCIS, Madrid, Spain). Irene Merino (Microbiology Service, Hospital Clinico de Salamanca, Instituto de Investigación Biomédica de Salamanca, Salamanca, Spain). Carla López-Causapé (Microbiology Service, Hospital Universitari Son Espases, Palma de Mallorca, Spain). Nuria Sabé and Evelyn Shaw (Infectious Diseases Service, Hospital Universitario de Bellvitge- Institut d'Investigacio Biomedica de Bellvitge, Barcelona, Spain). Dámaris Berbel and Fe Tubau 
Quintano (Microbiology Service, Hospital Universitario de Bellvitge- Institut d'Investigacio Biomedica de Bellvitge, Barcelona, Spain). Carlos Sánchez Carrillo and Emilia Cercenado (Microbiology Service, Hospital Gregorio Marañón, Madrid, Spain). Elena Sendra and Verónica Rubio (Infectious Diseases Service, Hospital de la Santa Creu i Sant Pau, Barcelona, Spain). Alba Rivera (Microbiology Service, Hospital de la Santa Creu i Sant Pau- Institut d'Investigació Biomèdica Sant Pau. Universitat Autònoma de Barcelona, Barcelona, Spain). Esther Calvo and Cristina Badía (Infectious Diseases Service, Hospital Mutua de Terrassa, Barcelona, Spain). Mariona Xercavins (Microbiology Service, Hospital Mutua de Terrassa, Barcelona, Spain). Ana de Malet (Microbiology Service, Hospital Marqués de Valdecilla, Santander). Luis Canoura-Fernández (Microbiology Service; Complexo Hospitalario de Pontevedra, Pontevedra, Spain). Soledad Salvo (Microbiology Service; Hospital Clínico Lozano Blesa, Zaragoza, Spain). Jose Ramón Paño-Pardo (Infectious Diseases Service; Hospital Clínico Lozano Blesa, Zaragoza, Spain). Francisco Carmona-Torre (Infectious Diseases Service; Clinica Universidad Navarra, Pamplona, Spain).

Funding. This study and the journal's Rapid Service Fee are sponsored and funded by MSD Spain. The study was also supported by Plan Nacional de I + D + i 2013-2016 and Instituto de Salud Carlos III, Subdirección General de Redes y Centros de Investigación Cooperativa, Ministerio de Economía, Industria y Competitividad, Spanish Network for Research in Infectious Diseases (REIPI RD16/0016/0004, RD16/ 0016/0005, RD16/0016/0007, RD16/0016/0010, RD16/0016/0011 and RD16/0016/0015), co-financed by the European Development Regional Fund 'A way to achieve Europe' (ERDF), Operative program Intelligent Growth 2014-2020.

Authorship. All named authors meet the International Committee of Medical Journal Editors (ICMJE) criteria for authorship for this article, take responsibility for the integrity of the work as a whole, and have given their approval for this version to be published.
Authors' Contributions. Conceptualization of the study: JPH, PRG and SG-Z. Study coordination: SG-Z. Data collection and investigation: SG-Z, FBA, ILM, IG, VP, BP, NB, LBP, MCF, MP, MRG, JAM, EMR, JLDP, AS, DG, IM, CLC, NS, ES, DB, FTQ, CSC, EC, ES, VR, AR, EC, CB, MX, AM, LC-F, SS, JRPP, FCT. Microbiological studies: FBA, ERG, RC, AO and PRG. Statistical analysis: SG-Z and XDJ. Writing original draft: SG-Z, FBA and ILM. Supervision: JPH, RC, AO and PRG. Review and edited the final manuscript: IG, VP, PB, NB, LBP, MCF, MP, MRG, JAM, EMR, JLDP, JDR, DLM, RC, AO, PRG, JPH.

List of Investigators. We thank the ITUBRAS-2 investigators who made this study possible. The REIPI and GEIRAS-GEMARA SEIMC ITUBRAS-2 group members: Silvia Gómez-Zorrilla, Inmaculada López Montesinos and Juan Pablo Horcajada (Infectious Diseases Service, Hospital del Mar, Institut Hospital del Mar d'Investigacions Mèdiques, Universitat Autònoma de Barcelona, CEXS-Universitat Pompeu Fabra, Barcelona, Spain); Federico BecerraAparicio, Desiré Gijón, Rafael Cantón and Patricia Ruiz-Garbajosa (Servicio de Microbiología, Hospital Universitario Ramón y Cajal and Instituto Ramón y Cajal de Investigación Sanitaria (IRYCIS) Madrid, Spain); Enrique Ruiz de Gopegui, Carla López-Causapé and Antonio Oliver (Microbiology Service, Hospital Universitari Son Espases, Palma de Mallorca, Spain); Inmaculada Grau, Nuria Sabé and Evelyn Shaw (Infectious Diseases Service, Hospital Universitari de Bellvitge- Institut d'Investigacio Biomedica de Bellvitge, Barcelona, Spain); Vicente Pintado (Infectious Diseases Service, Hospital Universitario Ramón y Cajal, Madrid, Spain); Belén Padilla (Infectious Diseases Service, Hospital Universitario Gregorio Marañón, Madrid, Spain); Natividad Benito, Elena Sendra and Verónica Rubio (Infectious Diseases Service, Hospital de la Santa Creui Sant Pau-Institut d'Investigació Biomèdica Sant Pau. Universitat Autònoma de Barcelona, Barcelona, Spain); Lucía Boix-Palop, Cristina Badía and Esther Calvo (Infectious Diseases Service, Hospital Mutua de Terrassa, Barcelona, Spain); Maria Carmen Fariñas (Infectious Diseases Service, Hospital Universitario Marqués de Valdecilla, 
Santander, Spain); María Peñaranda (Infectious Diseases Service, Hospital Universitari Son Espases, Palma de Mallorca, Spain); Maria Rocío Gamallo (Infectious Diseases Service, Complexo Hospitalario Universitario de Pontevedra, Pontevedra, Spain); Jose Antonio Martinez (Infectious Diseases Service, Hospital Clinic, Barcelona, Spain); Elena Morte-Romea and Jose Ramón Paño-Pardo (Infectious Diseases Service, Hospital Clínico Universitario "Lozano Blesa", Zaragoza, Spain); Jose Luis Del Pozo and Francisco Carmona-Torre (Infectious Diseases Service, Clínica Universidad de Navarra, Pamplona, Spain); Ana Siverio (Microbiology Service, Hospital del Mar, Institut Hospital del Mar d'Investigacions Mèdiques, Barcelona, Spain); Irene Merino (Microbiology Service, Hospital Clinico de Salamanca, Instituto de Investigación Biomédica de Salamanca, Salamanca, Spain); Dámaris Berbel and Fe Tubau Quintano (Microbiology Service, Hospital Universitario de Bellvitge- Institut d'Investigacio Biomedica de Bellvitge, Barcelona, Spain); Carlos Sánchez Carrillo and Emilia Cercenado (Microbiology Service, Hospital Gregorio Marañón, Madrid, Spain); Alba Rivera (Microbiology Service, Hospital de la Santa Creu i Sant Pau- Institut d'Investigació Biomèdica Sant Pau. Universitat Autònoma de Barcelona, Barcelona, Spain); Mariona Xercavins (Microbiology Service, Hospital Mutua de Terrassa, Barcelona, Spain); Ana de Malet (Microbiology Service, Hospital Marqués de Valdecilla, Santander); Luis Canoura-Fernández (Microbiology Service; Complexo Hospitalario de Pontevedra, Pontevedra, Spain) and Soledad Salvo (Microbiology Service; Hospital Clínico Lozano Blesa, Zaragoza, Spain).

Prior Presentation. These data were previously presented, in part, at the European Society of Clinical Microbiology and Infectious Diseases, 13-16 April 2019, Amsterdam, The Netherlands.

Disclosures. Juan Pablo Horcajada has received honoraria as speaker and for advisory boards from Pfizer, MSD, Menarini, Angelini and Zambon. Jazmin Diaz-Regañón and Diego López-Mendoza are employees of MSD Spain.
Silvia Gómez-Zorrilla, Federico Becerra-Aparicio, Inmaculada López-Montesinos, Enrique Ruiz de Gopegui, Inmaculada Grau, Vicente Pintado, Belén Padilla, Natividad Benito, Lucía Boix-Palop, Maria Carmen Fariñas, María Peñaranda, Maria Rocío Gamallo, Jose Antonio Martinez, Jose Luis Del Pozo, Xavier DuránJordá, Rafael Cantón, Antonio Oliver and Patricia Ruiz-Garbajosa report no potential conflicts. Elena Sendra is currently working in the Infectious Diseases Service, Hospital del Mar, Institut Hospital del Mar d'Investigacions Mèdiques, Barcelona, Spain. Veronica Rubio is currently working in the Hospital Municipal de Badalona, Barcelona, Spain.

Compliance with Ethics guidelines. Protocol and informed consent form were approved by the Clinical Research Ethical Committee of Hospital del Mar (registration number 2016/6957/I) and by the local Ethics Committees of each institution (information in Supplementary Material). Prior to initiation the study, an informed consent form was signed by the patient or guardian/legal representative. The study was conducted in accordance with the International Conference on Harmonization Good Clinical Practice Guideline and with the 1964 Helsinki Declaration and its later amendments or comparable ethical standards.

Data Availability. The datasets generated during and/or analyzed during the current study are available from the corresponding author on reasonable request.

Open Access. This article is licensed under a Creative Commons Attribution-NonCommercial 4.0 International License, which permits any non-commercial use, sharing, adaptation, distribution and reproduction in any medium or format, as long as you give appropriate credit to the original author(s) and the source, provide a link to the Creative Commons licence, and indicate if changes were made. The images or other third party material in this article are included in the article's Creative Commons licence, unless indicated otherwise in a credit line to the material. If material is not included in the article's Creative Commons licence and 
your intended use is not permitted by statutory regulation or exceeds the permitted use, you will need to obtain permission directly from the copyright holder. To view a copy of this licence, visit http://creativecommons.org/licenses/by$\mathrm{nc} / 4.0 /$.

\section{REFERENCES}

1. European Centre for Disease Prevention and Control. Point prevalence survey of healthcare-associated infections and antimicrobial use in European acute care hospitals 2011-2012. https://ecdc. europa.eu/sites/portal/files/media/en/publications/ Publications/healthcare-associated-infectionsantimicrobial-use-PPS.pdf. Accessed 9 July 2021.

2. Magill SS, O'Leary E, Janelle SJ, et al. Changes in prevalence of health care-associated infections in US hospitals. N Engl J Med. 2018;379:1732-44. https://doi.org/10.1056/NEJMoa1801550.

3. Mazzariol A, Bazaj A, Cornaglia G. Multi-drug-resistant Gram-negative bacteria causing urinary tract infections: a review. J Chemother. 2017;29:2-9. https://doi.org/10.1080/1120009X.2017.1380395.

4. Rodríguez-Baño J, Gutiérrez-Gutiérrez B, Machuca I, Pascual A. Treatment of infections caused by extended-spectrum-beta-lactamase-, AmpC-, and carbapenemase-producing enterobacteriaceae. Clin Microbiol Rev. 2018;31(2):e00079-17. https://cmr. asm.org/content/31/2/e00079-17.

5. Horcajada JP, Montero M, Oliver A, et al. Epidemiology and treatment of multidrug-resistant and extensively drug-resistant Pseudomonas aeruginosa infections. Clin Microbiol Rev. 2019;32:e00031e119.

6. Thaden JT, Fowler VG, Sexton DJ, Anderson DJ. Increasing incidence of extended-spectrum $\beta$-lactamase-producing Escherichia coli in community hospitals throughout the Southeastern United States. Infect Control Hosp Epidemiol. 2016;37:49-54.

7. Pérez-Vazquez M, Oteo-Iglesias J, Sola-Campoy PJ, et al. Characterization of carbapenemase-producing Klebsiella oxytoca in Spain, 2016-2017. Antimicrob Agents Chemother. 2019;63(6):e02529-18. https:// aac.asm.org/content/63/6/e02529-18.

8. Larramendy S, Deglaire V, Dusollier P, et al. Risk factors of extended-spectrum beta-lactamases-producing Escherichia coli community acquired urinary tract infections: a systematic review. Infect Drug Resist. 2020;13:3945-55.
9. Kelly AM, Mathema B, Larson EL. Carbapenem-resistant Enterobacteriaceae in the community: a scoping review. Int J Antimicrob Agents. 2017;50: 127-34.

10. Oliver A, Mulet X, López-Causapé C, Juan C. The increasing threat of Pseudomonas aeruginosa highrisk clones. Drug Resist Update. 2015;21-22:41-59. https://doi.org/10.1016/j.drup.2015.08.002.

11. Garner JS, Jarvis WR, Emori TG, Horan TC, Hughes JM. CDC definitions for nosocomial infections, 1988. Am J Infect Control. 1988;16:128-40.

12. Friedman ND, Kaye KS, Stout JE, et al. Health careassociated bloodstream infections in adults: a reason to change the accepted definition of community-acquired infections. Ann Intern Med. 2002;137:791-7.

13. Kollef $\mathrm{MH}$, Zilberberg MD, Shorr AF, et al. Epidemiology, microbiology and outcomes of healthcare-associated and community-acquired bacteremia: a multicenter cohort study. J Infect. 2011;62:130-5.

14. Smithson A, Ramos J, Bastida MT, et al. Differential characteristics of healthcare-associated compared to community-acquired febrile urinary tract infections in males. Eur J Clin Microbiol Infect Dis. 2015;34:2395-402. https://doi.org/10.1007/s10096015-2493-8.

15. Cheong HS, Kang C-I, Kwon KT, et al. Clinical significance of healthcare-associated infections in community-onset Escherichia coli bacteraemia. J Antimicrob Chemother. 2007;60:1355-60. https://doi. org/10.1093/jac/dkm378.

16. Shaw E, Benito N, Rodríguez-Baño J, et al. Risk factors for severe sepsis in community-onset bacteraemic urinary tract infection: Impact of antimicrobial resistance in a large hospitalised cohort. J Infect. 2015;70:247-54. https://doi.org/10.1016/j. jinf.2014.09.011.

17. Aguilar-Duran S, Horcajada JP, Sorlí L, et al. Community-onset healthcare-related urinary tract infections: comparison with community and hospital-acquired urinary tract infections. J Infect. 2012;64:478-83.

18. Horcajada JP, Shaw E, Padilla B, et al. Healthcareassociated, community-acquired and hospital-acquired bacteraemic urinary tract infections in hospitalized patients: a prospective multicentre cohort study in the era of antimicrobial resistance. Clin Microbiol Infect. 2013;19:962-8.

19. Charlson ME, Pompei P, Ales KL, MacKenzie RC. A new method of classifying prognostic in 
longitudinal studies: development. J Chronic Dis. 1987;40:373-83.

20. McCabe WRJG. Gram negative bacteremia. I. Etiology and ecology. Arch Intern Med. 1962;110: 845-7.

21. Rhee JY, Kwon KT, Ki HK, et al. Scoring systems for prediction of mortality in patients with intensive care unit-acquired sepsis: a comparison of the PITT bacteremia score and the acute physiology and chronic health evaluation II scoring systems. Shock. 2009;31:146-50.

22. The European Committee on Antimicrobial Susceptibility Testing. Breakpoint tables for interpretation of MICs and zone Version 9.0, 2019. http:// www.eucast.org. Accesss 10 July 2021.

23. Magiorakos AP, Srinivasan A, Carey RB, et al. Multidrug-resistant, extensively drug-resistant and pandrug-resistant bacteria: an international expert proposal for interim standard definitions for acquired resistance. Clin Microbiol Infect. 2012;18: 268-81. https://doi.org/10.1111/j.1469-0691.2011. 03570.x.

24. Jarlier V, Nicolas M-H, Fournier G, Philippon A. Extended broad-spectrum-lactamases conferring transferable resistance to newer-lactam agents in enterobacteriaceae: hospital prevalence and susceptibility patterns. Clin Infect Dis. 1988;10: 867-78. https://doi.org/10.1093/clinids/10.4.867.

25. Nayar R, Vivek Mohan Arora SD. Antibiotic impregnated tablets for screening ESBL and AmpC betalactamases. IOSR J Pharm. 2012;2:207-9.

26. Nordmann P, Poirel L, Dortet L. Rapid detection of carbapenemase-producing Enterobacteriaceae. Emerg Infect Dis. 2012;18:1503-7.

27. van Dijk K, Voets GM, Scharringa J, et al. A disc diffusion assay for detection of class A, B and OXA48 carbapenemases in Enterobacteriaceae using phenyl boronic acid, dipicolinic acid and temocillin. Clin Microbiol Infect. 2014;20:345-9.

28. Del Barrio-Tofiño E, López-Causapé C, Cabot G, et al. Genomics and susceptibility profiles of extensively drug-resistant pseudomonas aeruginosa isolates from Spain. Antimicrob Agents Chemother. 2017;61:1-13.

29. Kang C, Kim S, Park WB, et al. Bloodstream infections caused by antibiotic-resistant Gram-negative bacilli: risk factors for mortality and impact of inappropriate initial antimicrobial therapy on outcome. Antimicrob Agents Chemother. 2005;49(2): 760-6. https://doi.org/10.1128/AAC.49.2.760-766. 2005.
30. Baltas I, Stockdale T, Tausan M, et al. Impact of antibiotic timing on mortality from Gram-negative bacteraemia in an English district general hospital: the importance of getting it right every time. J Antimicrob Chemother. 2020. https://doi.org/10. 1093/jac/dkaa478/5997450.

31. Vardakas KZ, Rafailidis PI, Konstantelias AA, Falagas ME. Predictors of mortality in patients with infections due to multi-drug resistant Gram negative bacteria: the study, the patient, the bug or the drug? J Infect. 2013;66:401-14. https://doi.org/10.1016/j. jinf.2012.10.028.

32. López-Montesinos I, Domínguez-Guasch A, GómezZorrilla S, et al. Clinical and economic burden of community-onset multidrug-resistant infections requiring hospitalization. J Infect. 2020;80:271-8.

33. Friedman ND, Levit D, Taleb E, et al. Towards a definition for health care-associated infection. Open Forum Infect Dis. 2018. https://doi.org/10. 1093/ofid/ofy116/5001984.

34. Rodríguez-Baño J, López-Prieto MD, Portillo MM, et al. Epidemiology and clinical features of community-acquired, healthcare associated and nosocomial bloodstream infections in tertiary and community hospitals. Clin Microbiol Infect. 2009. https://doi.org/10.1111/j.1469-0691.2009.03089.x.

35. Lenz R, Leal JR, Church DL, Gregson DB, Ross T, Laupland $\mathrm{KB}$. The distinct category of healthcare associated bloodstream infections. BMC Infect Dis. 2012;12:85. https://doi.org/10.1186/1471-2334-1285.

36. European Centre for Disease Prevention and Control. Consumption of Antibacterials for systemic use (ATC group J01) in the community (primary care sector) in Europe. 2019. https://www.ecdc. europa.eu/en/antimicrobial-consumption/ database/rates-country. Accessed 14 Aug 2021.

37. Bell BG, Schellevis F, Stobberingh E, Goossens H, Pringle M. A systematic review and meta-analysis of the effects of antibiotic consumption on antibiotic resistance. BMC Infect Dis. 2014;14:13. https://doi. org/10.1186/1471-2334-14-13.

38. Gómez-Zorrilla S, Camoez M, Tubau F, et al. Antibiotic pressure is a major risk factor for rectal colonization by multidrug-resistant Pseudomonas aeruginosa in critically ill patients. Antimicrob Agents Chemother. 2014;58:5863-70.

39. Laupland KB, Church DL. Population-based epidemiology and microbiology of community-onset bloodstream infections. Clin Microbiol Rev. 2014;27:647-64. 
40. Weiner-Lastinger LM, Abner S, Edwards JR, et al. Antimicrobial-resistant pathogens associated with adult healthcare-associated infections: summary of data reported to the National Healthcare Safety Network, 2015-2017. Infect Control Hosp Epidemiol. 2020;41:1-18.

41. Vallés J, Rello J, Ochagavía A, Garnacho J, Alcalá MA. Community-acquired bloodstream infection in critically ill adult patients: impact of shock and inappropriate antibiotic therapy on survival. Chest. 2003;123(5):1615-24. https://doi.org/10.1378/ chest.123.5.1615.

\section{Publisher's Note}

Springer Nature remains neutral with regard to jurisdictional claims in published maps and institutional affiliations. 